\title{
Rebâtir après les défaites napoléoniennes : les enjeux de la reconstruction immobilière dans la France du Nord et de l'Est (1814-1860)
}

Jacques Hantraye

\section{(2) OpenEdition \\ Journals \\ Édition électronique \\ URL : https://journals.openedition.org/ahrf/9433 \\ DOI : 10.4000/ahrf.9433 \\ ISSN : 1952-403X \\ Éditeur : \\ Armand Colin, Société des études robespierristes}

Édition imprimée

Date de publication : 1 juin 2007

Pagination : 185-198

ISSN : 0003-4436

\section{Référence électronique}

Jacques Hantraye, «Rebâtir après les défaites napoléoniennes : les enjeux de la reconstruction immobilière dans la France du Nord et de l'Est (1814-1860) », Annales historiques de la Révolution française [En ligne], 348 | Avril-Juin 2007, mis en ligne le 01 juin 2010, consulté le 28 avril 2022. URL : http://journals.openedition.org/ahrf/9433; DOI : https://doi.org/10.4000/ahrf.9433

Ce document a été généré automatiquement le 28 avril 2022.

Tous droits réservés 


\title{
Rebâtir après les défaites napoléoniennes : les enjeux de la reconstruction immobilière dans la France du Nord et de l'Est (1814-1860)
}

\author{
Jacques Hantraye
}

1 " [...] À la place de l'hôtel de ville et du palais de justice, on ne voyait qu'un monceau de cendres et de décombres : les ruines de plus de deux cents bâtiments et de nombreux murs de jardins couvraient toutes les avenues de la ville; beaucoup d'autres édifices portaient les marques de la dévastation [...]. De toutes parts enfin, des murs sillonnés par les balles et les boulets attestaient les rudes alarmes auxquelles la population [...] avait dû être en proie au milieu de si vifs combats $»^{1}$. Cette description de Soissons après la chute du Premier Empire amène à s'interroger au sujet des destructions survenues durant la campagne de France, entre janvier et mars 1814, et de façon secondaire au cours de l'occupation qui commence pendant l'été 1815. Elle invite surtout à se pencher sur la « reconstruction » qui a suivi ainsi que sur l'effort consenti pour remettre en état les régions dévastées ${ }^{2}$. On n'envisagera ici que les aspects immobiliers de la question, dans les départements les plus touchés ${ }^{3}$.

2 Entre 1814 et le milieu de la décennie suivante, les populations demeurant près des anciens champs de bataille vivent souvent à proximité de ruines. L'ampleur des destructions est parfois telle que la perception de l'espace urbain est perturbée. À propos d'Huningue, très endommagée par les sièges de 1814 et 1815 , un voyageur note en 1816 qu'il a du mal à se frayer un chemin jusqu'à la place principale ${ }^{4}$. La même année, de passage dans la commune de Fouchères, un témoin écrit qu'il «marche sur les cendres et les ruines $»^{5}$. Même si un bilan général est difficile à établir, l'ampleur des dégâts est considérable, la première invasion étant de loin la plus destructrice. Le 29 mars 1814, les Prussiens mettent le feu au village de Venette : 160 maisons sont brûlées, 
les dommages sont évalués à 280000 francs $^{6}$. À Vendeuvre, sur 354 bâtiments d'habitation, 34 sont détruits, 96 inhabitables, et 280 bâtiments agricoles sont endommagés ${ }^{7}$. Les pertes totales à Nogent-sur-Seine s'élèvent à 2081977 francs, parmi lesquelles les destructions immobilières sont évaluées à $61 \%$, pour 133 maisons détruites et 239 dévastées en février $1814^{8}$. Des édifices prestigieux disparaissent. Le château de Pont-sur-Seine, belle construction du XVII ${ }^{e}$ siècle appartenant à la mère de l'empereur, est ainsi incendié en $1814^{9}$. Les données chiffrées concernant le département de l'Aube, certes particulièrement touché, offrent un aperçu plus synthétique. Le préfet indique en juillet 1814 que 2572 constructions ont été incendiées (44, $3 \%)$, tandis que 3234 autres ont été dévastées. Les édifices détruits par l'incendie représentent $71 \%$ de la valeur totale des pertes immobilières, qui est de 10858751 francs ${ }^{10}$. La somme est élevée car les destructions par le feu sont souvent complètes. En proportion, les villes souffrent davantage. Dans les arrondissements d'Arcis et de Nogent, le nombre des édifices endommagés au chef-lieu représente respectivement 15 et $36 \%$ de l'ensemble. Si les régions envahies souffrent indéniablement d'une atonie économique qui entrave le redressement, les conséquences démographiques sont difficiles à évaluer ${ }^{11}$. Le département de la Marne n'aurait perdu que $1,1 \%$ de sa population entre 1806 et $1821^{12}$. Mais Venette, dans l'Oise, ne compterait plus que 799 habitants en 1821, contre 852 en $1806^{13}$. Toutefois, il faut comparer les dégâts aux risques qui existent en temps de paix. L'Oise connaît ainsi au moins un incendie par mois entre 1815 et 1830 .

3 Après les destructions vient la phase souvent longue du déblaiement. À Soissons, les vestiges de l'hôtel de ville et du tribunal incendiés en 1814 ne disparaissent qu'en $1822^{14}$. À Nogent, où les autorités souhaitent en finir en 1819 avec les «ruines affligeantes, insalubres [et] dangereuses », les restes d'une construction occupée par la ville ne sont abattus qu'entre 1826 et $1829^{15}$. Les particuliers accomplissent cette tâche dans leurs propriétés, récupérant ce qui peut l'être. Pendant l'été 1814, on entreprend "de décombrer les matériaux des masures $»^{16}$ incendiées de Jacques Blampignon, à Méry-sur-Seine. On ne retrouve à cette occasion qu'un peu de ferraille, estimée à 18 francs ${ }^{17}$. L'hébergement des particuliers s'organise de façon provisoire. À Brienne, après l'incendie, les habitants apeurés s'installent dans les bois, en dépit du froid. À Nogent-sur-Seine, des habitants se construisent des abris parmi les ruines ${ }^{18}$. Ceux qui en ont la possibilité trouvent refuge ailleurs, par exemple chez leurs enfants ${ }^{19}$. Quand cela est possible, on transfère le siège des institutions dans un immeuble épargné. À Soissons, la mairie est installée dans l'ancien hôtel de l'intendance construit à la fin du XVIII ${ }^{e}$ siècle, qui fut acquis par la ville en $1834^{20}$. Les administrations de Nogent furent hébergées dans divers lieux entre 1814 et $1825^{21}$. Dans un premier temps, le conseil municipal et le tribunal campent dans les dépendances de l'hôtel-Dieu, avant que le second ne soit placé dans le nouveau palais de justice achevé en $1825^{22}$. Pour ce qui est de la mairie, la situation s'éternise pendant 45 ans $^{23}$. Les communes pâtissent parfois de ces déplacements. À Huningue, en 1816, les habitants aisés ont quitté la ville et le bureau de poste a été déplacé ${ }^{4}$.

4 L'esthétique des ruines fascine les voyageurs, à un moment où s'épanouit le goût pour les monuments du passé. L'Anglais Edward Stanley réalise ainsi en juillet 1814 un dessin représentant Berry-au-Bac ravagé25. Les murs des constructions particulières rappellent que les civils ont souffert. Encore visibles à Chaumont en 1856 ou à Nogent en 1859, ces traces sont perçues non pas comme des preuves de la violence de l'adversaire, mais de la volonté des Français de se défendre ${ }^{26}$. La monstration des ruines 
est moins un parti pris que le résultat d'une esthétique de hasard, liée à l'incertitude concernant le sort des édifices détruits. À Arcis-sur-Aube, par exemple, la façade du château, abîmée lors des combats de mars 1814, est restée en l'état vers 1848. L'édifice " est devenu un sujet de vénération pour les militaires qui passent à Arcis », écrit un historien de la localité. Des graffiti encouragent les générations futures à la valeur militaire, suivant l'exemple des combattants de la campagne de France ${ }^{27}$. En l'absence d'édifices commémoratifs, les ruines constituent des supports pendant le temps où se construit - au propre et au figuré - une mémoire des guerres de l'Empire. À la fin de la période, des monuments existent, qui rendent inutile le maintien des vestiges endommagés ${ }^{28}$. Il faut d'ailleurs mettre un terme à une situation pénible. À Nogent-surSeine, un pont de bateaux est aménagé au printemps de 1814, mais de multiples désagréments conduisent à la réalisation d'un pont en pierre vers $1830^{29}$. Enfin, il ne faut pas négliger le risque de délitement social. En 1816, un témoin note que "la population d'Huningue se compose maintenant de quelques centaines de malheureux qui, n'ayant ni pain, ni travail, semblent [...] destinés à la contrebande ou condamnés au brigandage $»^{30}$. Il convient donc d'envisager des solutions pérennes, ce qui passe par la recherche de fonds.

5 La question financière est complexe. L'autofinancement est le cas le plus commun. Le mobilier de l'église Saint-Pierre de Bar-sur-Aube ayant brûlé, les paroissiens se cotisent pour le remplacer ${ }^{31}$. Dans cette société du manque, le réemploi est une habitude, notamment lors de la reconstruction des églises. À Songeons, dans l'Oise, les matériaux de la mairie détruite en 1815 viennent en déduction du montant des travaux ${ }^{32}$. On vend également des matériaux, comme à Nogent ${ }^{33}$. Des pratiques de récupération sont attestées lors de la cession de biens détruits. En 1814, un charron se réserve un tas de tuiles à Méry, tandis qu'un propriétaire des environs de Montmirail garde des briques pour son usage ${ }^{34}$. Les municipalités doivent prendre à leur charge les fonds nécessaires à la reconstruction des édifices publics. C'est la réponse donnée dès 1828 par l'administration parisienne au sujet de l'hôtel de ville de Nogent, d'un coût de 10500 francs $^{35}$. La municipalité de Songeons emprunte pour rebâtir la mairie entre 1819 et $1822^{36}$. Les aides sont versées aux individus, et non en fonction de projets. Ainsi, le souspréfet de Nogent-sur-Seine se voit refuser la somme qu'il réclame pour réparer l'église et reconstruire l'hôtel de ville ${ }^{37}$.

6 La reconstruction s'accompagne d'un remaniement partiel de la propriété dont l'ampleur reste à évaluer. L'étude des minutes d'une étude notariale de Méry pour l'année 1814 permet de mettre en évidence quelques éléments qui n'ont qu'une valeur indicative ${ }^{38}$. Plus du tiers des ventes s'effectue au sein des familles. Les contractants sont proches géographiquement et socialement. Il s'agit d'artisans, de marchands et de cultivateurs. Les vendeurs sont souvent dans une situation de fragilité : sur treize personnes, on relève en effet six veuves et une malade. Les ventes, qui concernent la plupart du temps des bâtiments détruits dans leur totalité, sur des terrains d'une superficie inférieure à un hectare, commencent peu de temps après la fin des combats, sans doute par besoin de liquidités. Onze des quatorze contrats sont conclus en mai et en juin 1814. Les grands procèdent parfois de même. Le domaine de Pont-sur-Seine, propriété de Madame Mère depuis 1805, est vendu le 4 septembre 1814 à des acquéreurs parisiens ${ }^{39}$. Ces cessions sont parfois vécues douloureusement ${ }^{40}$.

7 En l'absence de système d'assurances, les prélèvements et les destructions liés à la guerre font dans le meilleur des cas l'objet de procédures de remboursement ou 
d'indemnisation de la part de l'administration. Le pouvoir a pris en compte la question des moyens financiers nécessaires à la reconstruction, ce qui n'est pas entièrement nouveau. Mettons à part l'implication directe de l'état dans la restauration des fortifications, pour des raisons stratégiques. C'est le cas à Soissons à partir de $1818^{41}$. Rappelons la tradition d'intervention du pouvoir auprès des victimes d'incendies ${ }^{42}$. Les sinistrés de la Restauration peuvent aussi solliciter le Bureau des incendiés de l'Aube, organisme public créé avant l'invasion ${ }^{43}$. Il répartit par exemple 26500 francs au printemps de 1815 "provenant des quêtes et de représentations données à Paris au profit des Incendiés par les événemens de la guerre $»^{44}$. Sous l'Ancien Régime déjà, le pouvoir a aidé les victimes de dommages de guerre ${ }^{45}$. Il est aussi intervenu sous l'Empire, à la suite des destructions de la Révolution ${ }^{46}$. La chronologie des procédures de remboursement et d'indemnisation est complexe. Elles commencent pendant les combats, afin de se concilier les populations. À Nogent, le 7 février 1814, il semble que Napoléon accorde « une large indemnité » aux habitants des maisons qu'il fait détruire pour la défense du lieu ${ }^{47}$. Après les combats, les chefs militaires alliés suivent ses traces, sans doute pour des raisons plus pratiques que politiques. Ainsi, le 9 mai, le comte de Raigecourt, gouverneur de la Haute-Marne au nom de l'Autriche, fait distribuer du bois à Chaumont pour réparer les maisons dévastées ${ }^{48}$. Le pouvoir royal envisage aussi une aide matérielle. À Châlons-sur-Marne, le 1er juin, le duc de Doudeauville, commissaire extraordinaire du roi dans la $2 \mathrm{e}$ division militaire, annonce que l'on va distribuer du bois pour réparer ou reconstruire les maisons, ainsi que les constructions publiques existant dans les campagnes, c'est-à-dire les églises, les presbytères et les ponts ${ }^{49}$. On estime que 384000 solives sont nécessaires dans le département de la Marne ${ }^{50}$. Ces attributions interviennent indépendamment de la reconstruction des bâtiments. À Aulnay-aux-Planches, dans la Marne, tout revient à des individus qui ont perdu des bâtiments, mais à Cormicy, 52 \% des solives vont à des artisans, pour l'exercice de leur état. On accorde avec parcimonie du bois pris en priorité dans les forêts communales ${ }^{51}$. Ces prélèvements ont contribué aux dégâts causés aux forêts. Une partie des secours versés en 1816 sont aussi gagés sur la vente de futaies de l'État ${ }^{52}$. Par ailleurs, les autorités constatent l'incapacité des habitants d'acquitter leurs impôts. En juillet 1814, 105 des 215 communes de l'arrondissement d'Épernay sont dans ce cas, d'où des exemptions. Le 3 juin 1814, on octroie du bois à des habitants de Champaubert et on leur remet le reliquat des contributions, vu « l'impossibilité de pouvoir faire face sans les secours du gouvernement $»^{53}$. Les voyages officiels sont l'occasion pour les grands de constater les dégâts et pour les populations d'exprimer leurs doléances. Le 8 septembre 1814 , le comte d'Artois inaugure un pont provisoire à Nogent-sur-Seine, en une mise en scène qui exprime l'espoir. Le prince s'enquiert des pertes, avant de donner 36000 francs aux nécessiteux ${ }^{54}$. Ceci est en rapport avec les premières mesures, comme l'ordonnance du 11 novembre $1814^{55}$.

8 Mais il faut envisager des actions de plus grande ampleur. Des enquêtes permettent d'évaluer l'ampleur des dégâts et de mesurer l'évolution de la situation. À Méry-surSeine, les pertes sont constatées dès le 27 juillet $1814^{56}$. La reprise de la guerre pendant les Cent-Jours relance le mouvement, sans doute pour des raisons politiques. Le 11 mai 1815, le ministre de l'Intérieur réclame ainsi au préfet de l'Aube des informations pour le cas où l'empereur agirait en faveur des sinistrés ${ }^{57}$. Dans ce même département, la procédure reprend de façon systématique au cours de la seconde occupation. À Rances, par exemple, l'enquête sur les dégâts de 1814 est menée par le juge de paix le 22 octobre $1815^{58}$. Les procès-verbaux dressent la liste des pertes et enregistrent 
l'évolution de la reconstruction, en incluant un questionnement sur la condition sociale des victimes. L'étude de l'espace permet en effet d'envisager des actions. À Soissons, à partir de 1816, on lève les plans des lieux détruits et on vérifie les états de pertes individuels. Les archives ayant disparu, on recense les habitants, en parallèle avec le nouveau numérotage des maisons ${ }^{59}$. À Arcis-sur-Aube, le plan cadastral est établi en 1827 , au cours de la reconstruction ${ }^{60}$.

9 Napoléon envisage d'aider les victimes de destructions par un décret du 6 avril $1815^{61}$. Les secours doivent être versés aux individus dont les habitations ont été détruites en 1814, en priorité les pauvres et les actifs. La chute de l'Empire met fin à ce projet et il faut attendre plusieurs mois pour trouver un texte comparable. Les ordonnances des 8 mai et 20 septembre 1816 affectent 11 millions de francs au «soulagement des départemens qui ont le plus souffert des calamités de la guerre ». Cette somme, qui équivaut à peu près au montant des destructions immobilières dans le seul département de l'Aube en 1814, est présentée comme un don de la famille royale ${ }^{62}$. Elle se compose de dix millions de francs retranchés de la liste civile du roi et de sa famille, et d'un million prélevé sur les sommes destinées au mariage du duc de Berry. L'ampleur des pertes conduit à réserver les secours à ceux qui ne pourraient réparer les dommages qu'ils ont subis, afin «de rebâtir leurs maisons incendiées ou démolies, remplacer les bestiaux, les meubles, les instrumens aratoires ou effets de première nécessité [...]». Ne sont admis à participer que les propriétaires payant moins de 200 francs de contribution foncière, "fermiers et artisans", mais aussi «marchands détaillants ». Là encore, il s'agit certes d'aider les pauvres, mais surtout des actifs disposant d'un minimum de biens.

10 La répartition effective varie suivant les départements, peut-être en fonction des pertes. Les quatre arrondissements de la Marne - sur cinq - ayant le plus souffert en 1814 se voient accorder 465500 francs sur les onze millions. L'écart est de un à neuf, Épernay bénéficiant de 67,5\% des secours et Châlons de 7, 5 \% seulement ${ }^{63}$. La Seine-etMarne reçoit pour sa part 350000 francs, la différence entre arrondissements étant ici de un à $2,4^{64}$. Les demandes sont examinées scrupuleusement. Dans la Haute-Marne, la commission chargée du remboursement des réquisitions réduit le total de $40 \%{ }^{65}$. Le maire de Méry prescrit pour sa part de donner moins à ceux à qui il est resté des matériaux ou qui ont reconstruit sur des structures existantes ${ }^{66}$.

11 La répartition est confiée à des administrateurs et à des notables. Dans chaque arrondissement, une commission de cinq membres choisis dans le conseil d'arrondissement, présidée par le sous-préfet, répartit l'argent entre les communes en consultant au besoin le contrôleur des contributions ${ }^{67}$. Au chef-lieu du département, la commission dirigée par le préfet est recrutée au sein du conseil général. Dans chaque commune, deux personnes distribuent l'argent sous la vigilance du maire. Elles peuvent faire appel au percepteur ${ }^{68}$. L'exemple de l'arrondissement de Senlis montre que l'on a pris soin de sélectionner des individus capables, honnêtes et impartiaux ${ }^{69}$. Le souspréfet d'Épernay recommande aux maires de répartir les sommes avec impartialité ${ }^{70}$. Le travail associe efficacité et volonté de susciter l'adhésion des populations au régime. À Montiéramey, de même qu'à Nogent-sur-Seine, dans l'Aube, ce sont le desservant et les autorités municipales qui répartissent l'argent en $1815^{71}$. La distribution des fonds s'accompagne dans certains cas d'un cérémonial qui suggère la renaissance du lieu. À Nogent, la distribution des cinq barils d'argent a lieu à la sous-préfecture en présence $\mathrm{du}$ préfet, après une messe ${ }^{72}$. En principe, les secours ne sont pas conditionnés par 
l'opinion politique des individus. En octobre 1816, un percepteur transmet des informations sur des individus susceptibles d'être secourus. Augustin Gauthier est décrit comme « intéressant par ses malheurs qu'il supporte avec résignation et sans se plaindre ». Quant à Edme Lambert, maréchal-ferrant, « [s]a conduite et ses sentimens politique n'inspirent aucune compassion pour lui » mais, précise la note, l'intention du roi et la justice veulent qu'il reçoive des secours ${ }^{73}$. La répartition revêt donc une dimension moralisatrice. Paradoxalement, au moment où sévit la Terreur légale, il s'agit aussi de mesures d'apaisement. Collectivités et particuliers n'en recherchent pas moins des passe-droits. La ville de Nogent, qui se recommande de la duchesse d'Angoulême, obtient ainsi 100000 francs sur les onze millions. La commission de l'arrondissement de Compiègne doit écarter des personnes aisées, inscrites à la suite de pressions $^{74}$.

Au-delà des intentions, il faut examiner les modalités de la répartition. Ces questions entraînent un travail administratif important. Dans la Haute-Marne, la commission chargée du remboursement des réquisitions se réunit 58 fois entre 1814 et $1817^{75}$. La commission de l'arrondissement de Compiègne pour la répartition des onze millions de francs siège pendant huit jours en octobre et novembre $1816^{76}$. Celles qui se réunissent en Seine-et-Marne en novembre 1816, dans un contexte de crise de subsistances, choisissent de maintenir la tranquillité publique en attribuant les secours aux plus pauvres, et non pas nécessairement aux exploitants, comme le prévoient les textes officiels, même si l'on tient compte des pertes dues à la guerre ${ }^{77}$. Ainsi, dans l'arrondissement de Provins, les communes qui paient le plus d'impôts reçoivent deux fois moins de secours que les autres. Le pragmatisme l'emporte donc. Généralement, le montant des secours est faible. À Margny-lès-Compiègne, Charles Paillot, dont les bâtiments ont été incendiés, reçoit $10 \%$ des 2000 francs de pertes qu'il déclare ${ }^{78}$. À Venette, les secours compris dans l'ordonnance de 1816 représentent $6 \%$ des sommes perdues en 1814 , sans compter il est vrai une distribution de bois ${ }^{79}$. L'État ne pouvait guère se montrer plus généreux dans le contexte budgétaire difficile des débuts de la Restauration, même si des compléments ont parfois été versés ultérieurement, parfois longtemps après ${ }^{80}$.

13 La répartition individuelle diffère selon les lieux : à Méry-sur-Seine, on privilégie un principe d'égalité - sans doute pour éviter les conflits -, tandis qu'à Barberey-SaintSulpice l'écart entre les individus est plus grand ${ }^{81}$. Des tensions ont pu naître, comme à Soissons, où les pertes mobilières furent plus indemnisées que les destructions $\mathrm{d}^{\prime}$ immeubles ${ }^{82}$. Sur le plan national, le caractère politique de l'indemnisation est le reflet de la rivalité entre Napoléon Ier et les Bourbons ${ }^{83}$. D'après le testament de l'empereur du 15 avril 1821, des sommes importantes sont censées revenir « aux villes et campagnes d'Alsace, de Lorraine, de Franche-Comté, de Bourgogne, de l'île-deFrance, de Champagne, Forez, Dauphiné, qui auraient souffert par l'une ou l'autre invasion ». Les villes de Brienne et de Méry, privilégiées, doivent recevoir un million chacune. Selon Jean Lemaire, Napoléon aurait voulu ainsi monter les habitants de ces régions contre le pouvoir royal, dont il pensait qu'il ne verserait pas les sommes en question, ce qui se confirma. Peu avant d'instituer la médaille de Sainte-Hélène, Napoléon III décide d'exécuter les volontés de son oncle. Brienne et Méry doivent toucher 400000 et 300000 francs, tandis que les 26 départements concernés reçoivent 50000 francs chacun. On achève de répartir les sommes en 1857. Elles servent à des 
œuvres de bienfaisance, mais à Brienne, par exemple, on construit aussi un hôtel de ville et on répare l'église.

La reconstruction n'est pas alors un phénomène nouveau. Des exemples passés attestent de la réussite possible de l'entreprise, tel Rennes au XVIII ${ }^{e}$ siècle. La Champagne a connu des épisodes de ce type, comme Arcis-sur-Aube après l'incendie de 1727. La reconstruction est aussi en lien avec la réparation des destructions révolutionnaires ou l'édification des villes neuves de Pontivy et La Roche-sur-Yon sous l'Empire ${ }^{84}$. Enfin, l'industrialisation entraîne la création de quartiers nouveaux, comme à Mulhouse sous la Restauration ${ }^{85}$. Les destructions survenues en 1814 permettent d'envisager des rénovations. À Soissons entre 1820 et 1825 un projet d'urbanisme prévoit l'aménagement de la voirie ${ }^{86}$. On relève également des transformations urbaines à Arcis-sur-Aube sous la monarchie constitutionnelle ${ }^{87}$. Mais la reconstruction se fait rarement ex nihilo, ce qui, outre le fait que seules des agglomérations de petite taille sont concernées, est sans doute à l'origine de l'oubli du phénomène par la mémoire collective. À Méry-sur-Seine, plusieurs individus ont reconstruit sur des murs subsistants ${ }^{88}$. Pourtant, le début des travaux put donner lieu parfois à des cérémonies marquantes. Le 11 juillet 1821, par exemple, le préfet de l'Aube se déplace à Nogent pour poser la première pierre de trois bâtiments édilitaires ${ }^{89}$.

On avait généralement peu construit d'édifices publics sous la Révolution et l'Empire. La reconstruction constitue donc une activité importante pendant plusieurs années, voire plusieurs décennies. Les travaux sont menés à un rythme discontinu, comme on le constate à Nogent. En 1818, la maison du bourrelier Edme Protat «est en reconstruction ». On va parfois plus lentement. En juin 1820, bien «que les fondations en sont faites depuis long tems [...], et les matériaux sur place», la maison du maçon Michel Bonhenry n'est toujours pas rebâtie, six ans après sa destruction ${ }^{90}$. L'édification de la gendarmerie et du palais de justice dure de 1821 à $1825^{91}$. À Rances, où 11 individus déclarent des pertes survenues en 1814, trois ont reconstruit en totalité et un à moitié, 19 mois après les faits. À Magny-Fouchard, commune elle aussi majoritairement peuplée de cultivateurs et dans une moindre mesure d'artisans, en janvier 1816, sur 17 cas précis, on recense une reconstruction à $10 \%$ de l'état antérieur, 5 à $33 \%, 5$ à $50 \%$, une à $60 \%$ et 5 à $75 \%$ environ, 22 mois après les destructions ${ }^{92}$. Des capitaux importants sont investis. En 1830, dans un contexte de crise économique, les travaux des fortifications de Soissons permettent d'employer la classe ouvrière ${ }^{93}$. À Nogent, en 1819, on souhaite que l'argent des travaux du tribunal soit dépensé entre des ouvriers recrutés sur place ${ }^{94}$. Toutefois, si l'élan est bien présent dans les villes, il n'existe pas forcément dans les espaces ruraux. À Magny et à Rances, la moitié des foyers n'a pu effectuer de travaux, principalement faute de moyens ${ }^{95}$. Sur 37 foyers de Magny, seuls deux ont des revenus fonciers leur permettant éventuellement de financer des travaux. Un ménage a vendu des terres et cinq ont emprunté pour rebâtir. À Rances, sept foyers ont d'autres propriétés dont le revenu est précisé, parmi lesquels deux peuvent en vivre. Des exploitations agricoles restent en friches, comme à Arsonval ${ }^{96}$.

La nouvelle configuration du bâti est quelque peu différente de ce qui existait avant la guerre, sans que l'urbanisme ou l'architecture soient radicalement changés. On profite de la remise en état des infrastructures pour opérer des améliorations. À Nogent, le pont est reconstruit à un autre emplacement, car la voirie a été modifiée ${ }^{97}$. Les projets dépendent des sommes que l'on peut y consacrer. À Méry, la veuve d'un boulanger qui 
possédait six maisons, deux granges et cinq écuries n'a édifié qu'une chambre en pierre. Au contraire, d'autres artisans et commerçants ont tout rebâti ${ }^{98}$. Dans les villes stratégiques, l'État fait réparer les fortifications, comme à Soissons ${ }^{99}$. L'enjeu majeur de la reconstruction est surtout la suppression des couvertures en chaume. Les circonstances poussent les autorités à accélérer cette transformation qui permet de lutter contre l'incendie ${ }^{100}$. Dès 1814 , le maire de Méry, souhaite "encourager la couverture en thuiles surtout [dans cette commune] ou les Batimens sont tres resserés $»^{101}$. Dans le canton de Compiègne entre 1806 et 1831 , les chaumières passent de 49 à $35 \%$ du total des maisons. À Venette, qui a particulièrement souffert des destructions, elles passent même de 79,5 à $28 \%^{102}$. La structure du bâti est parfois modifiée. Ainsi, à Brienne, au milieu du XIX ${ }^{\mathrm{e}}$ siècle, on «[...] construit de forts jolies maisons en pierres, tandis qu'autrefois elles étaient presque toutes bâties en bois [...] », ce qui va dans le sens de la diminution des causes d'incendie ${ }^{103}$.

17 La reconstruction oblige à définir les objectifs recherchés pour les nouvelles constructions. On les souhaite résistantes. À Châlons, le pont en pierre « [...] fut réparé en 1814 et n'a rien perdu de sa bonté ni de sa solidité $»^{104}$. On associe fonctionnalité et qualité esthétique. À Nogent, en 1819, on souhaite que les locaux du tribunal soient mieux distribués, tandis qu'en 1827 le conseil municipal attend un hôtel de ville " d'une grande simplicité », permettant une meilleure conservation des documents, et qui embellisse la ville ${ }^{105}$. Les édifices publics sont reconstruits dans le goût du temps. La mairie de Songeons est ainsi de style néo-classique ${ }^{106}$. Quant au nouveau château de Pont-sur-Seine, bâti «dans le genre italien", s'il ne se distingue pas "par son mérite architectural ", il contient des richesses historiques et artistiques, note un auteur en $1872^{107}$.

La reconstruction suscite enfin une interrogation identitaire qui apparaît dans les écrits concernant les villes reconstruites. Les bouleversements vécus génèrent un discours spécifique au sein du vaste courant d'histoire de la localité qui caractérise le XIX ${ }^{e}$ siècle. De la Monarchie de Juillet à la fin de la $\mathrm{II}^{\mathrm{e}}$ République se diffuse sous la plume d'érudits une évocation de la reconstruction qui ne se contente pas de célébrer le charme intemporel de la petite ville. Pour Lemaitre, ancien adjudant-major qui écrit vers 1840 à propos de Nogent, la reconstruction signifie renaissance et prospérité, tout en rappelant sans cesse l'événement originel qui place la cité sous le signe du combat patriotique ${ }^{108}$. L'identité urbaine est ambigüe, faite à la fois d'oubli et de valorisation d'un passé traumatique. Amédée Aufauvre, en 1859, ne mentionne pour sa part que l'effacement des traces de la destruction ${ }^{109}$. Cette idée est très répandue. Leroux écrit en 1839 qu' « on ne trouve à Soissons que des constructions modernes", or des édifices importants ont survécu aux destructions de 1814, ce qui montre que le bâti ancien n'est pas très valorisée ${ }^{110}$. Le discours est donc marqué par la surenchère, comme si l'on cherchait ainsi à effacer complètement les plaies. 1814 est considéré comme un point de départ. À Arcis, on insiste sur l'ordre et le progrès qui règnent désormais, les «constructions mieux bâties» dans une ville qui a "gagné en embellissement et en assainissement", ce que l'on dit aussi de Brienne ${ }^{111}$. La reconstruction implique davantage de sécurité et de qualité esthétique. En 1851, les dégâts dont l'hospice de Bar-sur-Aube eut à souffrir sont réparés « et le zèle des personnes qui y sont attachées promet une prospérité toujours croissante ${ }^{112}$. Ici comme à Brienne, on met l'accent de façon idyllique sur la solidarité. L'épisode aurait été surmonté grâce à une forte cohésion sociale, alors que l'on a pu constater que les tensions n'étaient pas absentes. Il 
ne semble pas que les villes rebâties aient été dénigrées par leurs habitants, sans doute parce que leur aspect évolue assez peu.

On a oublié cet épisode de reconstruction d'assez grande ampleur qui précéda l'ère haussmanienne. À des pertes moins conséquentes qu'au XXe siècle correspondent de faibles secours. Toutefois, il convient de noter que ceux-ci s'organisent dans la continuité de ce qui a été fait depuis la Révolution, en fonction d'enjeux politiques. L'État n'intervient encore que timidement dans des questions dont le règlement est confié avant tout aux municipalités et aux particuliers concernés. Cette étude, qui montre l'importance de l'épisode de 1814-1815, rappelle que ses séquelles s'inscrivent dans le temps long de l'histoire économique, sociale et culturelle de la France du XIX ${ }^{\mathrm{e}}$ siècle.

\section{NOTES}

1.LEROUX, Histoire de la ville de Soissons, t. 2, Soissons, Fossé Darcosse, 1839, p. 438-439.

2.On parle de « réparation » et surtout de « reconstruction » (AD Marne, 201 M 47, arrêté du commissaire extraordinaire dans la 2e division militaire, 1.6.1814), même si le maire de Nogent emploie en 1824 le mot « rétabli[ssement] » (AD Aube : 14 R 47, certificat du maire de Nogent, 15.3.1824).

3.L'étude concerne les départements suivants : Aisne, Aube, Haut-Rhin, Haute-Marne, Marne, Oise et Seine-et-Marne.

4.Anonyme, « Du trésor de Saint-Louis aux trois sièges d'Huningue », dans Société d'Histoire du Musée d'Huningue et du canton d'Huningue, 1957, p. 2-18, p. 13 et 16-17.

5.AD Aube, 14 R 41, percepteur de Virey-sous-Bar au sous-préfet de Bar-sur-Seine, 18.10.1816.

6.Louis GRAVES, Précis statistique du canton de Compiègne arrondissement de Compiègne, Paris, Guénégaud, 1983 (1855), p. 171.

7.AD Aube, 14 R 42, adjoint et conseil municipal de Vendeuvre, janvier 1815.

8. Madeleine TARTARY, Épisode de la Campagne de France. Nogent-sur-Seine en 1814, Paris, Les Presses Modernes, 1939, p. 173.

9.Arsène THÉVENOT, Histoire de la ville et de la châtellenie de Pont-sur-Seine, Paris, Le Livre d'histoire, 2004 (1873), p. 49.

10.AD Aube, 14 R 41, sd (1814-1815 ?), état des destructions dans l'Aube. On se situe entre les incendies localisés (Rennes perd 945 bâtiments dans l'incendie de 1720, cf Georges DuBY, dir., Histoire de la France urbaine, t. 3, Paris, Seuil, 1981, p. 461) et les destructions massives du XX ${ }^{\mathrm{e}}$ siècle (450 000 logements sont détruits en 1914-1918, id., t. 4, 1983, p. 136).

11.AD Marne, 201 M 47, adjoint de Sézanne, 29.7.1814; 201 M 217, note à propos des pertes dues à la guerre de 1814. AD Aube, $14 \mathrm{R}$ 41, préfet de l'Aube, 20.4.1815.

12. Hélène BOUCHER, Paroisses et communes de France, Marne, Paris, CNRS, 1984, p. 37. 13.Louis GRAVES, op. cit., p. 38-39. 
14.Geneviève CORDONNIER, Soissons. Son histoire illustrée à travers ses rues, places, monuments et ses habitants, Le Coteau, Horvath, 1986, p. 108.

15.AD Aube, 2 O 2452, sous-préfet de Nogent, 4.2.1819. Amédée AufAuVRE, Histoire de Nogent-sur-Seine, Res Universis, 1992 (1859), p. 193.

16. Maisons tombées en ruine.

17.AD Aube, 23 U 9, 13.6.1814 et $2 \mathrm{E}$ 16/113, maître Thomas, notaire à Méry, 16.8.1814.

18.Bourgeois, Histoire des comtes de Brienne, Troyes, Anner-André, 1848, p. 239.

Madeleine TARTARY, op. cit., p. 142.

19.AD Haute-Marne, E dépôt 121, 7 H 13, déclaration de pertes de Ragot, 24.10.1816.

20.Geneviève CORDONNIER, op. cit., p. 116-117.

21.Amédée AUfAUVRe, op. cit, p. 194.

22. Madeleine TARTARY, op. cit., p. 184.

23.AD Aube, $4 \mathrm{~N}$ 113, note, s d (1819). 20 2452, préfet, 1.10.1814 ; sous-préfet de Nogent, 4.2.1819; extrait de délibérations du conseil municipal de Nogent, 3.4.1827 ; conseil municipal de Nogent, vers le 3.4.1819. Amédée AUfAUVRE, op. cit., p. 194. En comparaison, la reconstruction de Rennes s'étend de 1720 à 1763 (Georges DUBY, dir., op. cit., t. 3, p. 461).

24.Anonyme, « Du trésor de Saint-Louis aux trois sièges d'Huningue », art. cit., p.17. 25.Edward STANLeY, Un Anglais sur les traces de Napoléon Bonaparte (trad. H. Pichelin), Paris, La Vouivre, 2003, p. 81.

26.Émile JoliboIs, Histoire de la ville de Chaumont, Paris, la Tour Gile, 1995 (1856), p. 439. Amédée Aufauvre, op. cit., p. 294.

27.CAMUT-CHARDON, Histoire d'Arcis-sur-Aube, Paris, Res Universis, 1989 (1848), 107, p. 34. Cf. aussi BOURGEOIS, op. cit, p. 240.

28.Le monument de la bataille de Montmirail est inauguré en 1867 (Inauguration du monument commémoratif de la bataille de Montmirail et Marchais le 11 février 1867, Châlonssur-Marne, Martin, 1867).

29.Amédée AUfAUVR, op. cit., p. 324 et Madeleine TARTARY, op. cit., p. 184-185.

30.Anonyme, « Du trésor de Saint-Louis aux trois sièges d'Huningue », art. cit., p. 17.

31.Louis CHEVALIER, Histoire de Bar-sur-Aube, Bar-sur-Aube, l'auteur, 1851, p. 39.

32.AD Oise, 2 O p 21 639, extrait des délibérations de Songeons, 3.2.et 22.5.1818.

33.Amédée AufAuvre, op. cit., p. 193.

34.AD Aube, 2 E 16/113, 14.6.1814.

35.AD Aube, 2 O 2452, ministre secrétaire d'État à l'Intérieur, 9.10.1828 et ordonnance du 3.3.1834.

36.AD Oise, 2 O p 21 639, minutes du préfet de l'Oise, 9.6.1818 et 29.7.1818.

37. Madeleine TARTARY, op. cit., p. 182.

38.AD Aube, 2 E 16/113, 1814.

39.Arsène THÉVENOT, op. cit., p. 112-113.

40.AD Haute-Marne, E dépôt 121, 7 H 13, déclaration de pertes de Morel, sd (1816 ?).

41.LEROUX, op. cit., p. 454-457.

42.Cf. un exemple concernant la Seine-et-Marne en 1812-1813 (AD Seine-et-Marne, 1 PP 147, ministre de l'Intérieur, 23.1.1813 ; sous-préfet de Coulommiers, 9.12.1812 et 23.1.1813).

43.AD Aube, $14 \mathrm{R} 47$, certificat du maire de Nogent, 15.3.1824 ; prince de HohenloheBartenstein, 17.2.1814; certificat du maire de Nogent, 17.3.1824. 
44.AD Aube, $14 \mathrm{R}$ 64, mandats de paiement de Payens (1.5.1815) et d'Ailleville (14.4.1815).

45.Yann GuIMON, Octobre 1746. La descente anglaise à Quiberon, Rennes, l'auteur, 1996, p. 124-126.

46. Claude AllemAND-COSNEAU et alii, Clisson ou le retour d'Italie, Paris, Imprimerie nationale, 1990 , p. 150.

47.J.-M. Lemaitre, Combat de Nogent-sur-Seine, Nogent, librairie des Petites-Affiches, sd (1840?), p. 8.

48.AD Haute-Marne, E dépôt 121, 7 H 3, arrêté du 9.5.1814.

49.AD Marne, 201 M 47, arrêté du 1.6.1814. Ambroise de Larochefoucauld, duc de Doudeauville (1765-1841), rentré d'émigration en 1799, membre du conseil général de la Marne, ministre et pair de France. Philanthrope, il s'occupa d'œuvres charitables (M. BRUGUIÈRE, La première Restauration et son budget, Genève, Droz, 1969, p. 136)

50.AD Marne, $201 \mathrm{M}$ 217, note à propos des pertes de 1814 .

51.AD Marne, $201 \mathrm{M} 47$, état des solives à accorder aux habitants, 10.6.1814 ; arrêté du commissaire extraordinaire, 1.6.1814.

52.AD Aube, 14 R 41, mandat, 28.3.1816.

53.AD Marne, $201 \mathrm{M} 47$, état des communes, arrondissement d'Epernay, 30.7.1814 ; arrêté du commissaire extraordinaire, 3.6.1814.

54. Madeleine TARTARY, op. cit., p. 172-173.

55.F.-F. STEENACKERS, L'invasion de 1814 dans la Haute-Marne, Paris, Didier, 1868, p. 313.

56.AD Aube : $14 \mathrm{R}$ 52, état des pertes à Méry, 27.7.1814.

57.Ibidem, $14 \mathrm{R}$ 41, ministre de l'Intérieur, 11.5.1815.

58.Ibidem, 14 R 52, Rances, 22.10.1815.

59.LEROUX, op. cit., t. 2, p. 439. Geneviève CORDONNIER, op. cit., p. 25, 129 et 157.

60. CAMUT-CHARDON, op. cit., p. 38.

61.AD Aube, 14 R 41, ministre de l'Intérieur, 13.5.1815.

62.AD Marne, 201 M 217, ordonnance du 20.9.1816.

63.Ibidem, $201 \mathrm{M} 217$, secrétaire général du ministère des Finances, 7.2.1817.

64.AD Seine-et-Marne, 8 R 50, procès-verbaux de Meaux et de Provins, 10.11.1816.

65.F.-F. STEENACKERS, op. cit., p. 318.

66.AD Aube, 14 R 52, maire de Méry à Tholmé, 27.7.1814.

67.AD Oise, R P 1706, commission d'arrondissement de Compiègne, 25.10-4.11.1816. AD Seine-et-Marne, 8 R 50, Fontainebleau et Meaux, 10.11.1816.

68.AD Marne, 201 M 217, ordonnance du 20.9.1816.

69.AD Oise, R P 1706, liste de candidats, 2.10.1816.

70.AD Marne, 201 M 217, circulaire du sous-préfet d'Epernay, 16.12.1816.

71.AD Aube, 14 R 64, états de répartition de Montiéramey (28.4.1815) et de Nogent-surSeine (5.5.1815).

72.Madeleine TARTARY, op. cit., p. 182.

73.AD Aube, 14 R 41, percepteurs de Virey-sous-Bar (18.10.1816) et de Lantages

(17.10.1816).

74. Madeleine TARTARY, op. cit., p. 178. AD Oise, R P 1706, procès-verbal de la commission d'arrondissement de Compiègne, 25.10-4.11.1816.

75.F.-F. STEENACKERS, op. cit., p. 318.

76.AD Oise, R P 1706, procès-verbal de la commission de Compiègne, 25.10-4.11.1816. 
77.AD Seine-et-Marne, $8 \mathrm{R}$ 50, procès-verbal de répartition du 12.11.1816 ; procèsverbaux de Meaux (10.11.1816), de Melun (9.11.1816) et de Provins (10.11.1816).

78.AD Oise, R P 1706, procès-verbal, commission communale de Margny, 28.10.1816.

79.Ibidem, secours aux habitants de Venette, 31.10.1816. Louis GRAVES, op. cit., p. 171. En 1812, les sinistrés de Mouroux, en Seine-et-Marne, avaient reçu 11,25\% sur des pertes de 35540 francs (AD Seine-et-Marne, 1 PP 147, ministre de l'Intérieur, 23.1.1813 ; souspréfet de Coulommiers, 9.12.1812 et 23.1.1813).

80.LEROUX, op. cit., t. 2, p. 439-440.

81.AD Aube, 14 R 64, états de répartition de Barberey-Saint-Sulpice (23.4.1815) et de Méry (29.11.1815).

82.LEROUX, op. cit., t. 2, p. 439-440.

83.Jean LemAIRE, Le testament de Napoléon, Paris, Plon, 1975, p. 175-177, 181, 184-185, 194 et 196-197.

84. CAMUT-CHARDON, op. cit., p. 26-27 et 35-36.

85.Georges Duby, dir., op. cit., t. 3, p. 127 et 573.

86.Geneviève CORDONNIER, op. cit., p. 23.

87.CAMUT-CHARDON, op. cit. p. 35-36. LEROUX, op. cit., t. 2, p. 459-460.

88.AD Aube, 14 R 52, Méry (1814 ?).

89.Amédée AUfAUVRe, op. cit., p. 169.

90.AD Aube, $14 \mathrm{R}$ 47, certificat du maire de Nogent, 2.4.1818; Bonhenry au bureau des incendiés, 20.6.1820.

91.Amédée AufAUVRe, op. cit., p. 169.

92.AD Aube, 14 R 52, tableaux, Rances, 22.10.1815 et Magny-Fouchard, 24.1.1816.

93.LEROUX, op. cit., t. 2, p. 458.

94.AD Aube, 2 O 2452, sous-préfet de Nogent, 4.2.1819.

95.Ibidem, 14 R 52, tableaux, Magny-Fouchard, 24.1.1816, et Rances, 22.10.1815.

96.Ibidem, $14 \mathrm{R} 41$, Matinot au préfet, sd, 1815-1816?

97.Dominique PRÉVOT, Les ponts de Nogent sur Seine, Nogent-sur-Seine, l'auteur, 2000, p. 38.

98.AD Aube, 14 R 52, Méry (1814 ?).

99.LEROUX, op. cit., t. 2, p. 454-457. S H D, M R 1177, rapport du 20.7.1846.

100.L'idée d'amélioration du bâti est déjà attestée lors de la reconstruction de Rennes (Georges DuBY, op. cit. t. 3, p. 462 et 466).

101.AD Aube, 14 R 52, maire de Méry, sd, environ 27.7.1814.

102.Louis GRAVES, op. cit., p. 50.

103.BOURGEOIS, op. cit., p. 244.

104.S H D, 1 M 1184, rapport de janvier 1825.

105.AD Aube, 2 O 2452, sous-préfet de Nogent, 4.2.1819; extrait des délibérations du conseil municipal de Nogent, 3.4.1827 ; conseil municipal de Nogent, s d, environ 3.4.1819.

106.Laurence MiRoux, L'Oise sous la Restauration (1815-1830), Beauvais, Gemob, 1990, photo $\mathrm{n}$ p. AD Oise, 2 o p 21 639, extrait des délibérations de Songeons, 3.2.1818. 107.Arsène THÉVENOT, op. cit., p. 14-15 et 113.

108.J.-M. LEMAITRE, op. cit., p. 41-42. De nos jours encore, le fronton de l'hôtel de ville de Méry, bâti sous le Second Empire, met en scène d'un côté des ruines, de l'autre des bâtiments reconstruits associés au mot « Pax » et à deux dates : 1814 et 1859.

109.Amédée AUfAUVRE, op. cit., p. 302. 
110.LEROUX, op. cit., t. 2, p. 459. Geneviève CORDONNIER, op. cit., 239 p.

111. CAMUT-CHARDON, op. cit., p. 35.

112.L. CheVAlier, op. cit., p. 80 et BOURGEOIS, op. cit., p. 243-244.

\section{RÉSUMÉS}

L'objectif de cet article est de comprendre quels ont été les moyens mis en œuvre pour rebâtir les villes et les villages détruits lors de la campagne de France de 1814 et en 1815 , de quelle manière s'est opérée cette « reconstruction » et comment les agglomérations restaurées ont été perçues par les contemporains. Il s'agit d'évaluer les pertes immobilières en les replaçant dans leur contexte et en s'interrogeant sur les procédures de l'enquête concernant les destructions. Le déroulement de l'indemnisation et de la collecte des fonds seront également abordés. L'administration, mais aussi la famille royale, sont intervenus de façon importante dans le processus d'indemnisation des victimes de destructions. L'étude de la reconstruction impose de réfléchir aux choix architecturaux et urbanistiques, aux améliorations envisagées, au statut des ruines et à l'effort lié à la reconstruction. Cet article ne prétend pas faire le point sur la question car la synthèse concernant ces thèmes n'est pas encore possible, requérant un travail beaucoup plus long.

Rebuilding after the Napoleonic defeats : the reconstruction of properties in northern and eastern France (1814-1860). The purpose of this article is to understand the means used to rebuild the cities and villages destroyed during the French campaign of 1814, how this reconstruction was done, and how these reconstructed towns were perceived by contemporaries. The property losses will be evaluated and placed in their proper context, and in addition, the method of assessing these loses will be examined. The process of indemnification and gathering funds will also be discussed. The administration and the royal family intervened in an important way in the process of indemnifying the victims of these destructions. A study of the reconstruction invites reflections about architectural and urban choices, about projected improvements, about the status of ruins, and the efforts related to reconstruction. This article does not claim to exhaust this subject, for a synthesis about these themes is still not possible, requiring as it does much more work.

\section{INDEX}

Mots-clés : guerre, destructions, indemnisation, urbanisme, reconstruction immobilière

\section{AUTEUR}

\section{JACQUES HANTRAYE}

42 boulevard Picpus

75012 Paris

jacques.ha@free.fr 